This item was submitted to Loughborough's Research Repository by the author.

Items in Figshare are protected by copyright, with all rights reserved, unless otherwise indicated.

\title{
Actual PV module performance including spectral losses in the UK
}

PLEASE CITE THE PUBLISHED VERSION

http://dx.doi.org/10.1109/PVSC.2005.1488452

PUBLISHER

(c) IEEE

\section{VERSION}

VoR (Version of Record)

\section{LICENCE}

CC BY-NC-ND 4.0

\section{REPOSITORY RECORD}

Williams, Sheryl R., Thomas R. Betts, Pongpan Vorasayan, Ralph Gottschalg, and David Infield. 2019. "Actual PV Module Performance Including Spectral Losses in the UK”. figshare. https://hdl.handle.net/2134/8206. 
This item was submitted to Loughborough's Institutional Repository (https://dspace.lboro.ac.uk/) by the author and is made available under the following Creative Commons Licence conditions.

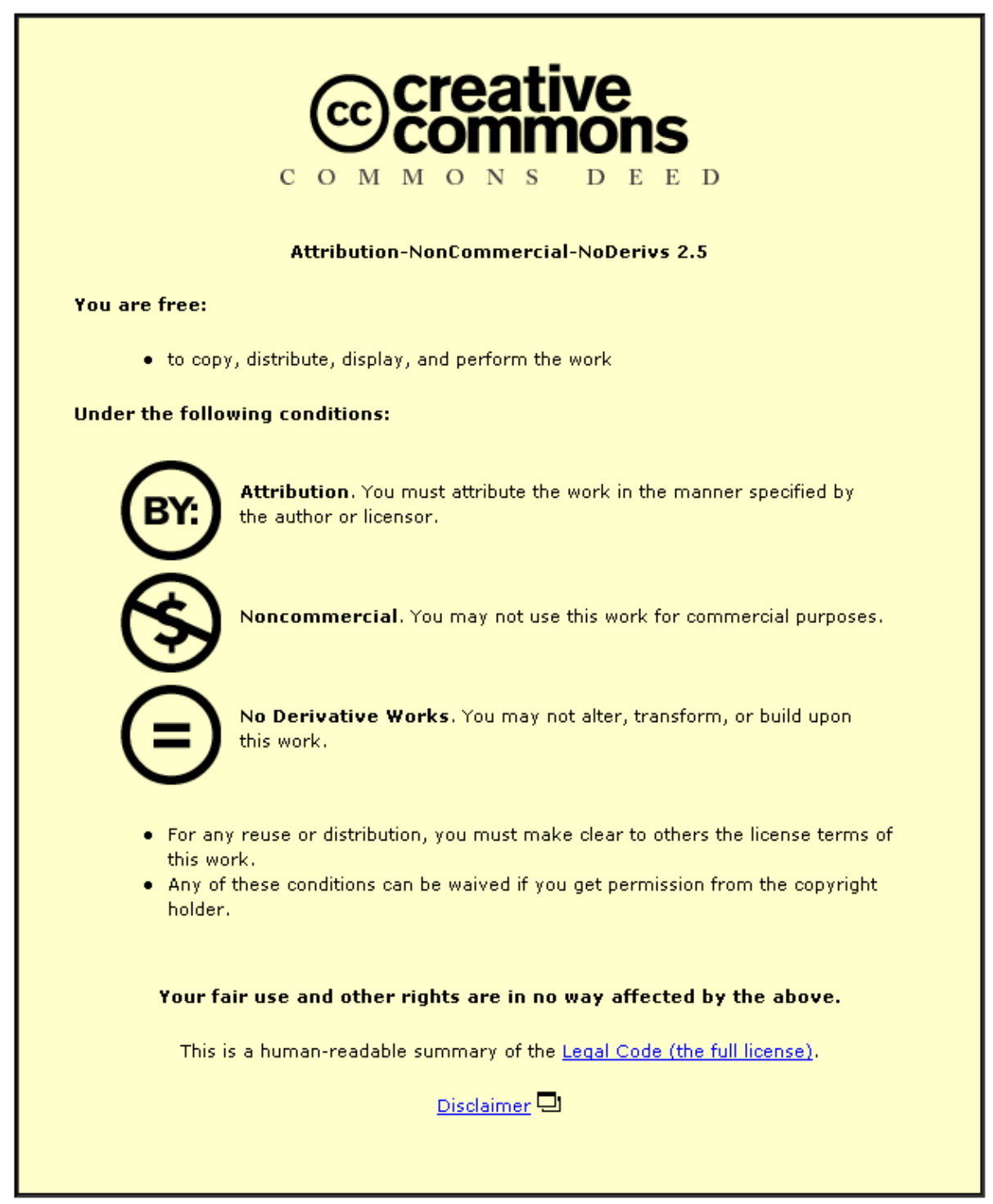

For the full text of this licence, please go to: http://creativecommons.org/licenses/by-nc-nd/2.5/ 


\title{
ACTUAL PV MODULE PERFORMANCE INCLUDING SPECTRAL LOSSES IN THE UK
}

\author{
S.R. Williams, T.R. Betts, P. Vorasayan, R. Gottschalg, D.G. Infield \\ Centre for Renewable Energy Systems Technology (CREST). Department of Electronic and Electrical \\ Engineering, Loughborough University, Loughborough, LE11 3TU, Tel: 01509-228148, Fax: 01509-610031, email: \\ S.R.Williams@lboro.ac.uk
}

\begin{abstract}
STC efficiencies are not sufficient to compare photovoltaic devices of different semiconductor material or device configurations. The energy yield will change as the variables of STC deviates from their original values when the modules are placed in various climatic conditions. The magnitude of this change for different modules is not always clear and needs to be investigated and modelled.

A modeling and analysis method named site specific conditions (SSC) is demonstrated which is a measure-correlate-predict approach. It allows an accurate estimation of the actual energy yield for different sites based on the measurements at one single site. The method takes into account the effect of the physical operating environment and translates this to other meteorological conditions on the basis of physics related formulae. Our results show a large seasonal variation for modules for the different effects. For crystalline modules losses of up to $12 \%$ in the summer is due to the temperature effect while the multi-junction thin film losses of more than $30 \%$ in the winter is due to spectral changes and incidence angle effect for the UK.
\end{abstract}

\section{INTRODUCTION}

The standard test conditions (STC) ${ }^{1}$ are used for laboratory and manufacturing quality testing. This rating is exclusively used for comparing different device technologies. However, under real, site-specific operating conditions operation close to STC is very rare. Any variation from STC will have an effect on device efficiency, which will in turn result in very different energy production than that stipulated by STC measurements. Indeed, it has been shown that modules with the same efficiency rating can have differences in the energy production by as much as $50 \%$ [1].

Each location has a specific operational environment where STC may or may not be encountered. As an example of the lack of relevance of STC for some sites, the frequency of these conditions is demonstrated exemplary for Loughborough, UK: In the year 2003 the combination of irradiance $1000 \mathrm{~W} / \mathrm{m}^{2}$ and module temperature of $25^{\circ} \mathrm{C}$ was seen only $0.29 \%$ of the daylight time. When including the spectrum, the relevance of these specific conditions is non-existent.

It is therefore imperative to develop a more realistic energy rating, which should be based on an understanding of the science governing the performance

${ }^{1}$ Irradiance $1000 \mathrm{~W} / \mathrm{m}^{2}$, module temperature of $25^{\circ} \mathrm{C}$, an AM1.5 spectrum and normal incidence variations and should include a methodology to translate the energy rating from one site to the energy predicted at another through simple calculations. This must be based on an understanding of the variability of divergent environmental conditions as well as their influence on device performance. The site specific conditions (SSC) presented here, is such an energy prediction method. It has the added advantage of disentangling the different environmental loss factors and thus lends itself also as an approach to analyze specific effects associate to different locations.

To date, there are several performance models available, requiring different levels of input. Two of these models are specifically relevant to this work. One is the measure-correlate-predict method by Ransome et al. [2] where the maximum power performance of a system in one site is used to predict the performance of the same system in another site. This method is very accurate but amalgamates some environmental effects and thus is not suitable to evaluate site specific measurements. The second method is the RRC by Raicu et. al [3] where the indoor characterized module performance coefficients are used to translate to real efficiency at a site with its meteorological data.

The model presented in this work, SSC, uses the strength of both of these approaches to generate a methodology not only able to separate different environmental effects from each other but allows the prediction of the actual energy yield in $\mathrm{kWh} / \mathrm{kWp}$ to a high accuracy. The model is applied to measurements taken at the Centre for Renewable Energy Systems Technology (CREST) first to demonstrate the importance of different environmental effects and then to demonstrate the accuracy of the approach.

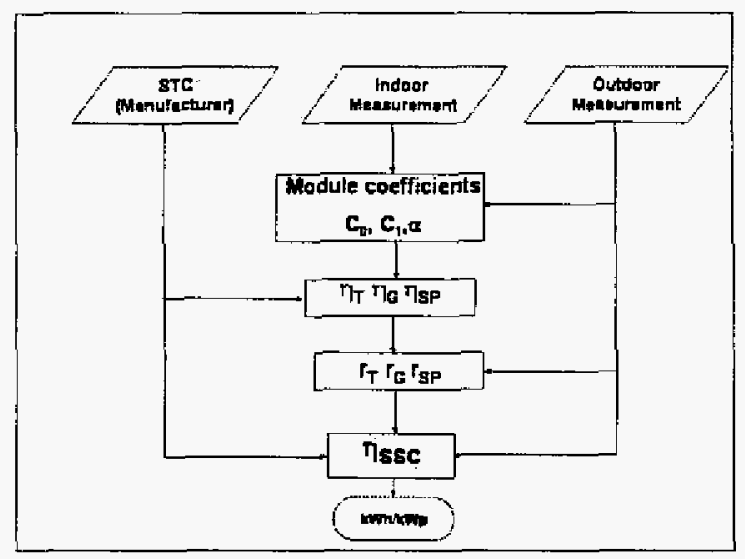

Figure 1: Aл algorithm of the procedure of SSC 


\section{PERFORMANCE MODEL}

In this work the RRC method [3] has been modified in order to utilize outdoor-measurement based data. The basic idea of this model is to model the effect of different operating conditions independent of each other through the following equation.

$$
\eta_{S S C}=r_{T} \cdot r_{G} \cdot r_{S P} \cdot r_{L A} \cdot r_{X} \times \eta_{S T C}
$$

Where the terms are defined as follows: $n_{s s c}$ is the SSC efficiency; $n_{s T c}$ is the STC efficiency; $r_{Y}$ is the factor which describes the effect of each variable as they deviate form STC: $T$ is temperature; $G$ is irradiance different; SP is spectrum; IA is incidence angle; $X$ is for other is inverter efficiency or any further influence to be considered.

The 0ssc provides the actual operating efficiency over a specified time period, which can be shown to be equivalent to the irradiance-weighted mean efficiency as in equation 2

$$
\eta_{S S C}=\frac{\sum \eta \cdot G}{\sum G}
$$

The $0_{\text {ssc }}$ can then be used to calculate the actual energy yield in terms of $\mathrm{kWh} / \mathrm{kWp}$ as in equations 3 .

$$
\text { EnergyYield }=\frac{k W h(\text { incident })}{k W p(S T C)} \times \eta_{S S C}
$$

The systematic approach algorithm is summarized in Figure 1. Each STC variable is evaluated in turn while the others are held constant. The resulting effects are then combined as in equation 1 to obtain a value for Ossc.

The basic approach of how to extract the separate parameters required for the calculations was described in [4]. There is, however, a major improvement applied in this paper, in that the spectrally corrected irradiance $G_{\text {corn, }}$ which is defined as the irradiance corrected to the AM 1.5 spectral distributions, is described as given below, rather than based on the useful irradiance used in the previous paper:

$$
G_{c o r r}=\frac{\frac{I_{S C}}{G}}{\frac{I_{S C}(S T C)}{G(S T C)}} \times G
$$

The data used for the analysis presented in this work comprises of a range of data set measured at CREST's test facility. The test procedure and measurement system are described in [5] The modules studied employ a variety of currently available commercial technologies such as amorphous, a-Si, (single, double and triple junctions), polycrystalline (p-Si) and mono-crystalline(c-Si) silicon, copper-indiumdiselenide (CIS). Their measurement period range from 6 months to over 3 years. Further, higher powered devices are currently in the initial testing period and data will be presented on future conferences. Other devices, such as CIGS and CdTe are measured but have not yet fulfilled a fuli operational year and are thus not included in the analysis here.

\section{RESULTS AND DISCUSSION}

\section{Annual and Seasonal Variation}

Figure 2 shows the seasonal variation of the real efficiency for 5 different modules operation at CREST during 2003. The values are relative to the nameplate efficiency of the each module. There is a significant difference in the device behavior between the different technologies. The reasons for this will be explained in the following, demonstrating the use of the SSC calculations. The largest variation of actual efficiency from winter to summer (nearly $20 \%$ ) is seen for the a-Si modules. The reasons for these performance variations are due to disparate responses to different environmental factors. These environmental factors are unfortunately often correlated and not easily separated. A case in point is the higher irradiance in summer that will almost always be associated with high module operating temperature. Thus temperature and irradiance can be intertwined. Furthermore, the low air mass results in bluer spectra, which then again results in the misinterpretation of a positive temperature for some devices.

The variation of the monthly operating efficiency is shown in Figure 2 and as expected, the efficiency of the $\mathrm{c}-\mathrm{Si}$ and $\mathrm{p}-\mathrm{Si}$ devices is twice as high as that of the thin film devices. More interestingly, the seasonal trends exhibited by the two different materials are directly opposite.
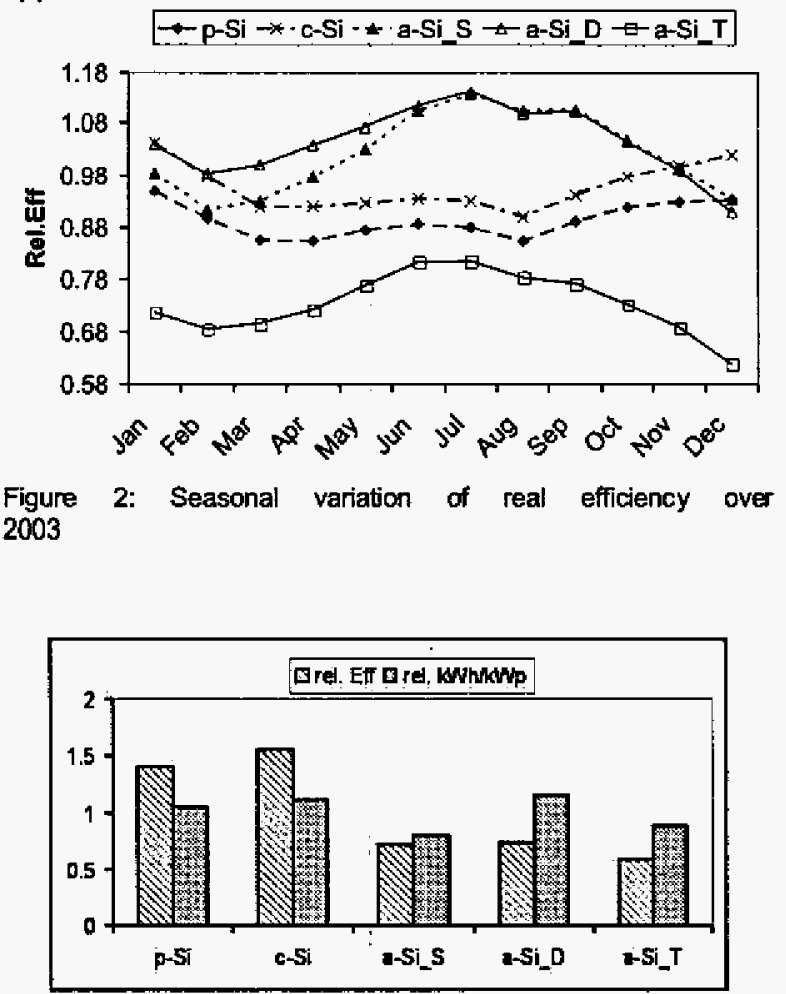
A commonly used measure of merit for devices and systems is the energy yield in $\mathrm{kWh} / \mathrm{kWp}$ Figure 3shows the values for the annual efficiency and $k W h / k W p$ for the modules for 2003, where the values are relative to the group-average. The crystalline devices outperform thin film ones when the comparison is based on efficiency. On the other hand, when using $\mathrm{kWh} / \mathrm{kWp}$ as the basis for comparison, the double junction outperforms the crystalline by almost $10 \%$. Care must however be taken when using the $\mathrm{kWh} / \mathrm{KWp}$, as pointed out by [2], since it depends overly on the declared power and thus underrating as done by few manufacturers significantly skews any such results.

For both annual and seasonal performance comparison the accurate identification and quantification of individual effects is beneficial. The method presented here is applied in the following for an estimation of the importance of the different environmental effects.

\section{Temperature Effect}

The effect of increased temperature measured in the laboratory is universally a decrease in performance. This is shown in figure 4 , where the spectrally uncorrected and corrected temperature coefficients, as derived from the measurements, are discussed. The influence of temperature on a-Si modules appears less clear, as the temperature coefficient extracted from the spectrally uncorrected irradiance measurements is positive but if the incident spectrum is considered, the temp-

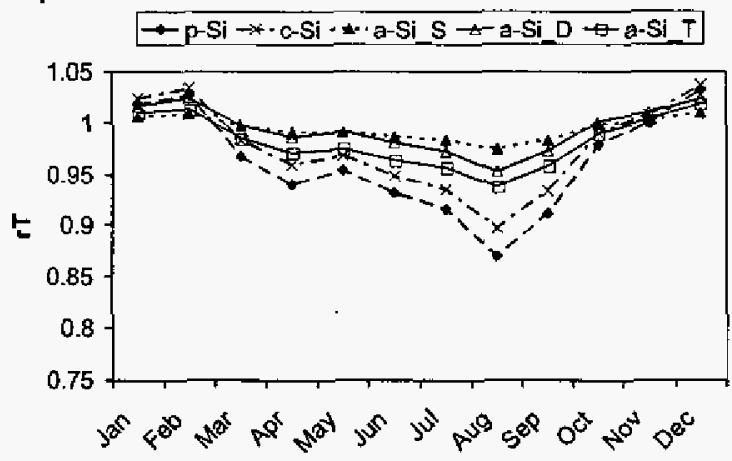

Figure 4: Seasonal variation of the temperature effect in 2003

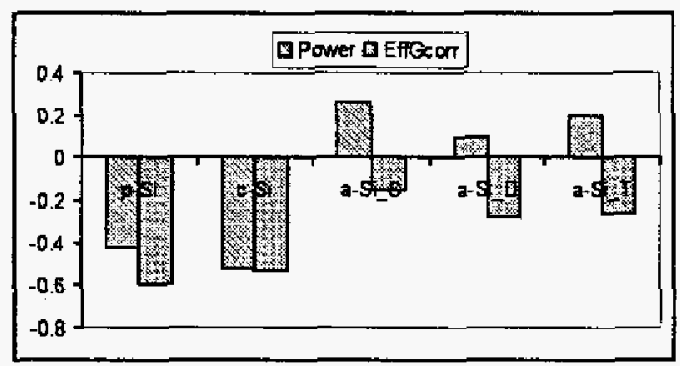

Figure 5: Temperature coefficients for tested modules in $\% / \mathrm{C}$

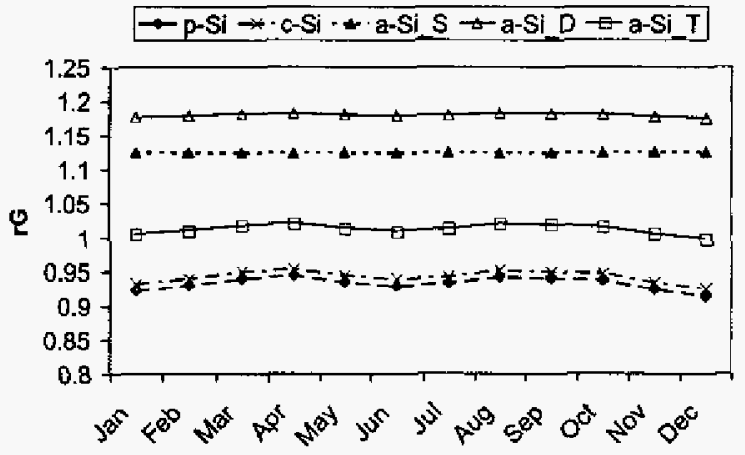

Figure 6: Seasonal variation of the irradiance effect in 2003 $\rightarrow-p-S i-* \cdot c-S i-a-S i s-a-S i+D+a-S i T$

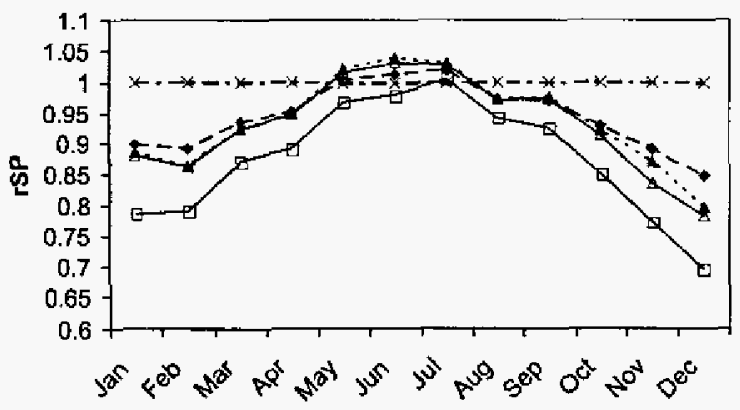

Figure 7: Seasonal variation of the spectral effect in 2003

erature coefficient becomes negative. Using spectrally corrected data allows the identification of the seasonal variation of the thermal losses, as done in Figure 4. As expected, the effect for a-Si is smaller than for c-Si. All devices have a small gain in winter, were the operating temperatures are below $25^{\circ} \mathrm{C}$.

\section{Irradiance Effect}

The effect of irradiance is dependent largely on parasitic resistances. In low irradiance conditions, the shunt resistance will dominate device performance while the high irradiance conditions are dominated by series resistance. While the c-Si type of devices exhibit an increase with irradiance intensity, this is not necessarily the case for thin film devices. The transparent conducting oxides used as a front contact have a specific resistivity which is one order of magnitude higher than that of a metal and thus the series resistance of these thin film devices will be typically higher. This results in a decline in efficiency of for high irradiances. The ideal performance is often found in the mid range irradiance values.

In this sense, one can say that the irradiance effect shown in Figure 6 is as expected because the irradiance effect calculated relative to the nameplate STC values is fairly consistent over the seasons. More surprising is the magnitude of this, but this is due to the normalization to nameplate efficiency rather than measured outdoor efficiency and thus is prone to variations in the production batch. This explains the very high values a-Si single and double junction. Furthermore both these devices have outstanding shunt resistances and thus their low light performance (with respect to STC) is inconsistency high.

\section{Spectral and Incidence Angle Effects}


It is more difficult to model the individual effect of spectral and incidence angle than that of temperature and irradiance. The approach chosen here is based on analyzing $I_{s c} / G$ rather than the efficiency and assuming the resulting effect to be independent of any other effect and linearly related to efficiency

Figure 7 investigates the seasonal spectrum. It is slightly surprising that the c-Si and p-Si benefit from the spectral conditions in winter time. Devices do not utilize all spectral irradiance equally well, and thus the conclusion is that the spectral match of these specific devices is better suited to winter spectra than summer spectra in Loughborough. The amorphous silicon devices have a distinct minimum in December, close to the shortest day and thus the time of the 'reddest' spectrum. The annual contribution can be slightly positive.

So far, the SSC was used for the analysis of device performance and generated some information on what is driving the performance of different devices. The results of the energy yield calculated by the combined SSC are then presented in Figure 8. The over-prediction of the SSC model for the multi-junction devices are due to reflection loses, which were not investigated in this paper in any detail. Overall, the results indicated that the $\mathrm{kWh} / \mathrm{kW}$ p values calculated with the SSC are within $10 \%$ of the actual measured values for most of the modules. The different of $19 \%$ seen for the triple junction will change when the reflection losses are accounted for.

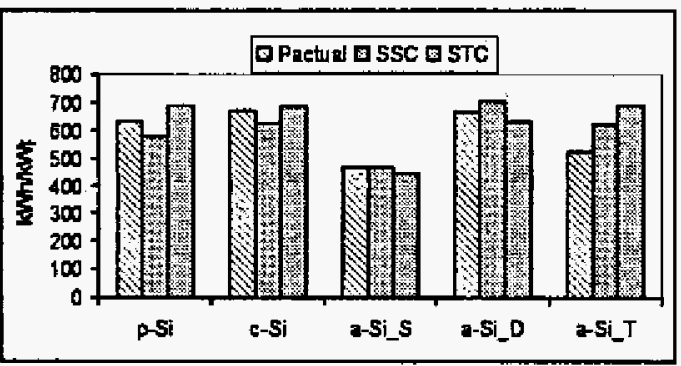

Figure 8: Annual energy production of the modules in 2003

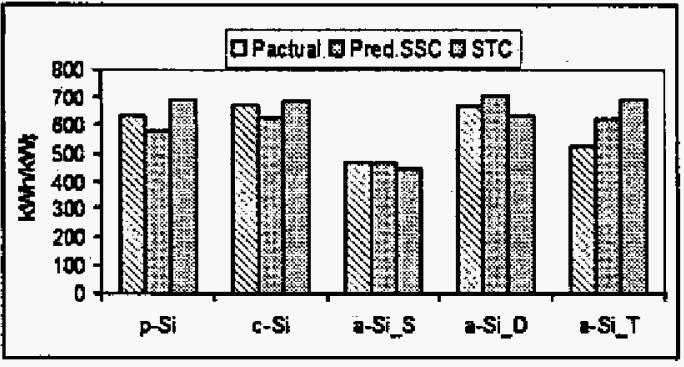

Figure 9: Annual energy production of the modules predicted for 2004.
It is clearly understood that the effect of incidence angle is two fold. The first relating to the reflection from different layers in the module structure and the impact this has on light propagation to the absorber layer(s). A ray tracing approach using Fresnel equations will be adopted suited for application to multi-junction. The second is the effect relating to the changing ratio of the beam and diffuse irradiance component, the differences in reflection mechanism for these fluxes and also the resulting impact on the spectrum the absorber layer sees. The measurement system needed for this analysis is currently being built and the result will be published in future works.

\section{Predicting with SSC}

It was shown that the SSC is well able to reproduce the measurements used to extract the underlying parameters, but that is not an independent test. In order to test the validity and accuracy of this model it was applied to independent sets of data. Here, sets from CREST's measurement campaign for different years for the same modules are used. It would be useful to use data from different climates but that will have to be done in the future. Figure 9 shows the predicted energy production for 2004 using the 0 ssc parameters extracted from the modules 2003 data. The predicted values show good agreement for several of the modules within tor$8 \%$. The a-Si triple junction value was almost $18 \%$ this we believe to be due to the large complex of the multijunction and how the effects like spectrum and incidence angle affect it. This requires further investigation.

\section{CONCLUSION}

A method to analyse outdoor data is presented, which is based solely on outdoor data. This SSC model was used to calculate the annual energy produced in 2003 and predict for 2004 with an error of + or $-8 \%$ for most of the modules. The a-Si triple junction error was significantly higher and this is believed to be the complexity of the effect of spectrum and incidence angle this will be address in future. Also the validity of this model for other location and data sets will be continued.

\section{Acknowledgements}

R. Gottschalg is funded through an EPSRC Advanced Research Fellowship (EPSRC GR/T03307/01).

\section{REFERENCES}

CN. Jardine, et a., " PV-Compare: Direct Comparison of Eleven PV Technologies at Two Locations in Northern and Southem Europe", $17^{\text {th }}$ EUPVSEC, 2001, pp724-727.

S. J. Ransome and J. H. Wohlgemuth, "Understanding and Correcting $\mathrm{kWh} / \mathrm{kWp}$ Measurements", PV in Europe, 2002, pp.

A. Raicu, et al., "Annual and Seasonal Energy Rating of mon-Si, a-Si and GaAs Test Cells for the USA by the RRC Method", IEEE PVSC, 1991, pp. 744-749.

S.R. Williams, et al., "Modeling Long-Tem Module Performance Based on Realistic Reporting Conditions with Consideration to Spectral Effects", 3rd WCPVSEC, 2003, pp.

S.R. Williams, et al., "Performance of Photovoltaic Modules in a Temperate Maritime Climate", $3^{\prime}$ WCPVSEC,2003.pp. 